\title{
DERECHO Y POLÍTICAS AMBIENTALES EN LA REGIÓN DE MURCIA
}

\author{
ELISA PÉREZ DE LOS COBOS HERNÁNDEZ \\ Profesora asociada de Derecho Administrativo \\ Universidad de Murcia \\ Abogada \\ SANTIAgo M. Álvarez CARREÑo \\ Profesor titular de Derecho Administrativo \\ Universidad de Murcia
}


Sumario: 1. Breve reflexión introductoria. 2. Manifestaciones de la política y legislación ambiental de la Administración regional en Murcia. 2.1. Premios de Desarrollo Sostenible de la Región de Murcia. 2.2. Control y vigilancia de las emisiones industriales a la atmósfera. 2.3. Estacionamiento en el Parque Regional de Calblanque, Monte de las Cenizas y Peña del Águila. 2.4. Medidas de prevención de incendios forestales en la Región de Murcia. 2.5. Agricultura. A. Programa de Desarrollo Rural de la Región de Murcia 2014-2020: ayudas para las medidas de agroambiente y clima y agricultura ecológica. B. Ayudas para la reconstitución del potencial de producción agrario. 2.6. Pesca. A. Obtención de datos e información sobre el estado de la flota pesquera artesanal. B. Horario especial de calamento de los artes de pesca en las zonas delimitadas para el baño en el Mar Menor. 2.7. Caza. 2.8. Sanidad animal. 3. Ordenanzas locales. 3.1. Cieza. A. Aprobación definitiva del Reglamento regulador del servicio público de abastecimiento de agua potable al municipio de Cieza (BORM, núm. 238, de 15 de octubre de 2015). B. Aprobación definitiva de la Ordenanza reguladora del uso y vertido de aguas residuales a la red de alcantarillado (BORM, núm. 238, de 15 de octubre de 2015). 3.2. Blanca: aprobación inicial de la modificación de la Ordenanza reguladora de la intervención administrativa en la edificación y uso del suelo (BORM, núm. 217, de 19 de septiembre de 2015). 3.3. Yecla: convocatoria de subvenciones en el ámbito de los programas de medio ambiente, ejercicio 2015 (BORM, núm. 174, de 30 de julio de 2015). 3.4. Lorquí: aprobación definitiva de la Ordenanza reguladora del uso y vertidos a la red de alcantarillado del municipio de Lorquí (BORM, núm. 170, de 25 de julio de 2015). 3.5. Mazarrón: modificación de la Ordenanza municipal de uso y aprovechamiento de playas (BORM, núm. 137, de 17 de junio de 2015).

\section{Breve reflexión introductoria}

Ciertamente, este segundo semestre del año 2015 no ha estado exento de actividad normativa en materia ambiental en la CARM. Parece como si el nuevo Gobierno, surgido de las pasadas elecciones autonómicas, se hubiera propuesto demostrar que, a pesar de la desorganización administrativa, la protección ambiental ocupa un puesto importante en su agenda. Son medidas menores, básicamente resoluciones y órdenes, de contenido ambiental indirecto o en sentido amplio (se trata más bien de medidas de salud pública o que tienden a preservar actividades de pesca o caza, etc.).

No se detecta desde luego ningún giro hacia una política ambiental verdaderamente seria que intente afrontar de manera radical amenazas tan graves para la Región de Murcia como el cambio climático, la desertización, la aparición cada vez más frecuente de especies invasoras, la contaminación del aire en poblaciones como Alcantarilla, etc.

En los momentos actuales, cuando los partidos ya se preparan para las próximas elecciones generales, estaremos atentos a los guiños o promesas ambientales, de las que tomaremos buena nota y de cuya falta de aplicación efectiva me temo que deberemos seguir dando cuenta en esta crónica a nuestros improbables lectores. 


\section{Manifestaciones de la política y legislación ambiental de la Administración regional en Murcia}

\subsection{Premios de Desarrollo Sostenible de la Región de Murcia}

Los premios en materia de medio ambiente tienen en la Región de Murcia un largo recorrido, convocándose por primera vez en el año 2002 con el nombre de Premios de Calidad Ambiental. En 2006, y recogiendo las recomendaciones contenidas en el Pacto Social por el Medio Ambiente, estos premios pasaron a denominarse Premios de Desarrollo Sostenible ${ }^{1}$. Estos premios se han visto reforzados por la Ley 4/2009, de 14 de mayo, de Protección Ambiental Integrada, que establece en su título VI, bajo la rúbrica "Fomento del medio ambiente y lucha frente al cambio climático", numerosas referencias a la necesidad de impulsar e incentivar desde la Administración regional determinados comportamientos y modelos de desarrollo sostenible. En atención a esto, la Consejería de Agricultura, mediante Orden de 28 de mayo de 2015, ha convocado la octava edición de los Premios de Desarrollo Sostenible de la Región de Murcia².

Estos premios tienen un doble objetivo: por una parte, reconocer públicamente el esfuerzo realizado por empresas, instituciones, organizaciones y colectivos de la Región de Murcia en materia de desarrollo sostenible; y, por otra, difundir aquellas experiencias de éxito e ideas que puedan ser referentes para otros. De acuerdo con esto, los premios integran diferentes modalidades: i) Premios de Desarrollo Sostenible: en los que se valoran los esfuerzos en gestión ambiental sostenible, ecoeficiencia e innovación ambiental, incluyendo, en cuanto a este último aspecto, tecnologías y procedimientos, productos y servicios, y métodos de gestión cuyo impacto sea menos perjudicial para el medio ambiente que otras alternativas disponibles, realizados por empresas y cualquier otro tipo de organización; ii) Premios a la Iniciativa frente al Cambio Climático: en los que se tienen en cuenta las iniciativas, los proyectos y las actividades cuyo objetivo sea la mitigación y/o adaptación a los impactos del cambio climático; iii) Premios a la Responsabilidad Social Corporativa en materia de Medio Ambiente: respecto a los proyectos, las actividades y los compromisos asumidos voluntariamente por las

\footnotetext{
${ }^{1}$ Vid. Protocolo General suscrito entre la Consejería de Industria y Medio Ambiente y las organizaciones representativas de la actividad económica y social de la Región de Murcia, para fomentar el desarrollo sostenible en el marco del Pacto Social por el Medio Ambiente, autorizado por el Consejo de Gobierno en sesión de 17 de noviembre de 2006 (BORM, núm. 20, de 25 de enero de 2007).

${ }^{2}$ Orden de 28 de mayo de 2015, de la Consejería de Agricultura, por la que se regula y convoca la octava edición de los Premios de Desarrollo Sostenible de la Región de Murcia (BORM, núm. 125, de 2 de junio de 2015).
} 
empresas y organizaciones empresariales en relación con el medio ambiente en el ámbito de la responsabilidad social corporativa; iv) Premios de Educación Ambiental: en los que se tienen presentes las iniciativas, los proyectos y las actividades que contribuyan a la generación y disposición de información para la educación ambiental y la sensibilización de empresas y ciudadanos; y, por último, v) Premio Mención Especial: que se otorgará tras valorar la trayectoria de ciudadanos, empresas y cualquier tipo de organización especialmente representativos en el campo del medio ambiente.

\subsection{Control y vigilancia de las emisiones industriales a la atmósfera}

Existe un profuso marco normativo destinado a evitar o aminorar los daños que puedan derivarse de la contaminación atmosférica para las personas, el medio ambiente y demás bienes de cualquier naturaleza. Dentro de este, las labores de prevención, vigilancia, control y reducción de la contaminación atmosférica resultan fundamentales ${ }^{3}$. En este sentido, el pasado 1 de julio de 2015, la Dirección General de Medio Ambiente aprobó las instrucciones técnicas en materia de control y vigilancia continua de emisiones industriales a la atmósfera ${ }^{4}$. En concreto, fueron aprobadas la Instrucción Técnica para el registro y transmisión automática de información relativa a la medición continua de emisiones y parámetros en las instalaciones (IT DGMA SPYEA-ATM-2.0.) y la

\footnotetext{
${ }^{3}$ En este sentido, destacan, entre otras normas, las siguientes: Decreto 833/1975, de 6 de febrero, por el que se desarrolla la Ley 38/1972, de 22 de diciembre, de Protección del Ambiente Atmosférico; Real Decreto 117/2003, de 31 de enero, sobre limitación de emisiones de compuestos orgánicos volátiles debidas al uso de disolventes en determinadas actividades; Real Decreto 653/2003, de 30 de mayo, sobre incineración de residuos; Real Decreto 430/2004, de 12 de marzo, por el que se establecen nuevas normas sobre limitación de emisiones a la atmósfera de determinados agentes contaminantes procedentes de grandes instalaciones de combustión, y se fijan ciertas condiciones para el control de las emisiones a la atmósfera de las refinerías de petróleo; Ley 34/2007, de 15 de noviembre, de Calidad del Aire y Protección de la Atmósfera; Orden ITC/1389/2008, de 19 de mayo, por la que se regulan los procedimientos de determinación de las emisiones de los contaminantes atmosféricos $\mathrm{SO}_{2}, \mathrm{NO}_{\mathrm{x}} \mathrm{y}$, partículas procedentes de las grandes instalaciones de combustión, el control de los aparatos de medida y el tratamiento y remisión de la información relativa a dichas emisiones; Resolución de 8 de julio de 2009 , de la Secretaría de Estado de Energía, por la que se precisan determinados aspectos relativos a la aplicación de la Orden ITC/1389/2008, de 19 de mayo, y de la Orden PRE/3539/2008, de 28 de noviembre, respecto a la remisión de información y al seguimiento y control de las emisiones a la atmósfera procedentes de las grandes instalaciones de combustión; Real Decreto 100/2011, de 28 de enero, por el que se actualiza el catálogo de actividades potencialmente contaminadoras de la atmósfera y se establecen las disposiciones básicas para su aplicación; Real Decreto 815/2013, de 18 de octubre, por el que se aprueba el Reglamento de Emisiones Industriales y de desarrollo de la Ley 16/2002, de 1 de julio, de Prevención y Control Integrados de la Contaminación; y Norma UNE-EN-14181:2005, incorporada como norma nacional el 23 de marzo de 2005 y adaptada recientemente, el 22 de abril de 2015, a su nueva versión UNE-EN-14181:2015.
}

${ }^{4}$ Resolución de 1 de julio de 2015, de la Dirección General de Medio Ambiente, por la que se aprueban instrucciones técnicas en materia de control y vigilancia continua de emisiones industriales a la atmósfera (BORM, núm. 178, de 4 de agosto de 2015). 
Instrucción Técnica para el aseguramiento de la calidad de los datos de los sistemas automáticos de medición (IT DGMA SPYEA-ATM-3.0). La adaptación de los sistemas automáticos de medición - ya instalados y convenientemente autorizados - a lo establecido en estas instrucciones técnicas se realizará en el plazo máximo que establezca la concreta autorización o, en su defecto, en el plazo máximo de un año a partir del 1 de julio de 2015.

\subsection{Estacionamiento en el Parque Regional de Calblanque, Monte de las Cenizas y} Peña del Águila

El espacio natural de Calblanque, Monte de las Cenizas y Peña del Águila fue declarado Parque Regional mediante la disposición adicional tercera de la Ley 4/1992, de 30 de julio, de Ordenación y Protección del Territorio de la Región de Murcia. Desde 1995, este espacio dispone de su propio Plan de Ordenación de los Recursos Naturales (PORN), aprobado por Decreto 45/1995, de 26 de mayo, cuyo artículo 99 dispone que “el acceso público al litoral mediterráneo del Parque podrá ser controlado, en el marco de la vigente Ley de Costas, por la Consejería de Medio Ambiente en los sectores y épocas que estime oportunos para la adecuada preservación de los recursos y sin perjuicio de las competencias que legalmente correspondan a los municipios"5. En atención a esto, durante los años de vigencia del PORN, las distintas consejerías competentes en materia de espacios naturales han adoptado los más diversos modelos de gestión, con resultados desiguales. Así, en los años 2010, 2011 y 2012 se aprobaron órdenes de vigencia temporal en los meses de julio y agosto para ordenar temporalmente el acceso de los vehículos a motor al interior del Parque, con el fin de contener la entrada excesiva de visitantes y evitar el deterioro de los hábitats naturales y de los hábitats de las especies.

En la actualidad, el Parque Regional forma parte del Área de Planificación Integrada núm. 8, Sierras de Cartagena, estando previstas la elaboración y aprobación del Plan de Gestión Integral (PGI) ${ }^{6}$. Dicho PGI incorporará el PORN y el Plan Rector de Uso y

\footnotetext{
${ }^{5}$ Decreto 45/1995, de 26 de mayo, por el que se aprueba el Plan de Ordenación de los Recursos Naturales de Calblanque, Monte de las Cenizas y Peña del Águila (BORM, núm. 151, de 1 de julio).

${ }^{6}$ Teniendo en cuenta que la planificación de los espacios naturales, regulada con carácter básico por la Ley 42/2007, de 13 de diciembre, del Patrimonio Natural y de la Biodiversidad, ha de realizarse de forma coordinada con los requerimientos de las directivas de Hábitats y de Aves, la Orden de 25 de octubre de 2012, sobre la planificación integrada de los espacios protegidos de la Región de Murcia, procede a
} 
Gestión que se elabore, y contemplará, entre otras cuestiones, las determinaciones precisas para una planificación global del uso público del Parque. Mientras tanto, para este año 2015 se ha hecho necesario establecer un modelo de control de accesos que busca garantizar el objetivo de conservación de los hábitats, contar con el apoyo social de todos los colectivos implicados y no suponer un perjuicio a las utilidades que los valores naturales ofrecen a sus propietarios privados. De acuerdo con esto, a propuesta de la Dirección General de Medio Ambiente, la Consejería de Agricultura y Agua dicta la Orden de 10 de junio de 2015, por la que se regula el acceso y estacionamiento, durante el periodo estival, de vehículos a motor en el Parque Regional de Calblanque, Monte de las Cenizas y Peña del Águila ${ }^{7}$. En esta se concreta que, durante los meses de junio a septiembre de 2015, el acceso de vehículos a motor debe realizarse mediante el dispositivo de ordenación de accesos establecido por la Dirección General de Medio Ambiente, con arreglo al cual, el Servicio de Planificación, Áreas Protegidas y Defensa del Medio Natural puede adoptar todas las medidas necesarias para que en ningún momento se supere la capacidad de carga del espacio protegido $^{8}$. Si bien debe tenerse en cuenta que, en ningún caso, las limitaciones de acceso acordadas pueden afectar a los propietarios de los terrenos, titulares de derechos sobre su uso, a los responsables del mantenimiento de los servicios públicos municipales o de vigilancia, o a las personas que participen en otros trabajos expresamente autorizados por la Dirección General de

integrar el Parque Regional dentro del Área de Planificación Integrada núm. 8, Sierras de Cartagena. De acuerdo con esto, se prevén la elaboración y aprobación del Plan de Gestión Integral, que se llevarán a cabo con arreglo al calendario establecido por los acuerdos de los consejos de Gobierno de 7 de junio y 6 de julio de 2013.

${ }^{7}$ Orden de 10 de junio de 2015, por la que se regula el acceso y estacionamiento, durante el periodo estival, de vehículos a motor en el Parque Regional de Calblanque, Monte de las Cenizas y Peña del Águila (BORM, núm. 136, de 16 de junio de 2015). Han de tenerse en cuenta las competencias para la gestión de los espacios naturales protegidos que tiene encomendadas la Consejería de Agricultura y Agua (Decreto del Consejo de Gobierno 42/2014, de 14 de abril, por el que se establecen los Órganos Directivos de la Consejería de Agricultura y Agua; Decreto de la Presidencia 4/2014, de 10 de abril, de reorganización de la Administración Regional; y Decreto 26/2011, de 25 de febrero, por el que se establece la estructura orgánica de la Consejería de Agricultura y Agua).

${ }^{8}$ Entre otras medidas, destacan las siguientes: i) el acceso de vehículos a motor se someterá a control mediante barreras; ii) se regulará el acceso de vehículos a motor en el período comprendido entre el 27 de junio y el 6 de septiembre, ambos incluidos, desde las $10.00 \mathrm{~h}$ hasta las $20.00 \mathrm{~h}$, hasta alcanzar la capacidad de acogida admisible de las áreas de estacionamiento, que se establece en 466 vehículos, momento en el cual el acceso a estas áreas quedará temporalmente prohibido; y iii) se delegan en la directora general de Medio Ambiente la modificación extraordinaria de la capacidad de carga máxima establecida en las áreas de estacionamiento por razones de conservación, y la variación de los horarios de acceso para su adaptación a las necesidades del dispositivo de ordenación. Además, los lunes del mes de septiembre $(7,14,21$ y 28) se pondrá en marcha la iniciativa "Los lunes sin coche", días en los que, aparte de proporcionar la información adecuada a los visitantes, no se permitirá el estacionamiento de vehículos a motor en ninguna área de las dispuestas al efecto, salvo para el acceso de personas con movilidad reducida/discapacidad. 
Medio Ambiente, que deben tener libre acceso al Parque Regional para el desarrollo de las actividades permitidas.

\subsection{Medidas de prevención de incendios forestales en la Región de Murcia}

De conformidad con lo dispuesto en la Orden de 24 de mayo del 2010, de la Consejería de Agricultura y Agua, sobre medidas de prevención de incendios forestales para el año 2010, queda prohibida, con carácter general, la realización de quemas agrícolas y forestales en la época de peligro ${ }^{9}$. Se exceptúan, previa autorización y salvo durante los meses de julio y agosto, aquellos casos en los que las quemas tengan por objeto prevenir o paliar daños causados por plagas o enfermedades, o evitar otros riesgos de mayor gravedad, todo ello bajo medidas extraordinarias de prevención. Asimismo, queda previsto que, cuando existan razones de interés social, condiciones meteorológicas especialmente desfavorables u otros motivos que así lo aconsejen, las autorizaciones expedidas pueden suspenderse temporalmente en su vigencia y efectos, hasta que desaparezcan dichas razones o motivos ${ }^{10}$.

Visto lo anterior, y teniendo en cuenta las especiales condiciones meteorológicas de este último año, el Servicio de Planificación, Áreas Protegidas y Defensa del Medio Natural ha elaborado un informe que justifica la suspensión temporal de la expedición de autorizaciones de quema por parte de los agentes medioambientales. Esta suspensión temporal no tiene efectos respecto de aquellas autorizaciones que la Dirección General de Medio Ambiente pudiera emitir al objeto de prevenir o paliar daños causados por plagas o enfermedades o evitar otros riesgos de mayor gravedad, bajo medidas extraordinarias de prevención. Asimismo, ante las adversas condiciones de sequía y estrés hídrico del combustible forestal - lo que favorece de manera importante la evolución negativa de un posible conato de incendio-, este año 2015 se ha propuesto una modificación en la concreción de la época de peligro.

Así, mediante Resolución de 5 de mayo, la Dirección General de Medio Ambiente acuerda ampliar, para el año 2015, la época de peligro, estableciéndola en el período

\footnotetext{
${ }^{9}$ Artículo 9 de la Orden de 24 de mayo del 2010, de la Consejería de Agricultura y Agua, sobre medidas de prevención de incendios forestales para el año 2010 (BORM, núm. 121, de 28 de mayo de 2010).

${ }^{10}$ Disposición adicional segunda de la Orden de 24 de mayo del 2010, de la Consejería de Agricultura y Agua, sobre medidas de prevención de incendios forestales para el año 2010.
} 
comprendido entre el 15 de mayo y el 30 de septiembre ${ }^{11}$; además, suspende, con carácter temporal, la vigencia y los efectos de las autorizaciones para quemas agrícolas y forestales emitidas sobre la base de la precitada Orden de 24 de mayo de 2010, de la Consejería de Agricultura y Agua, hasta que las condiciones meteorológicas desfavorables remitan; y, por último, suspende la vigencia y los efectos de las autorizaciones de uso del fuego en áreas recreativas o similares con combustible vegetal.

\subsection{Agricultura}

A. Programa de Desarrollo Rural de la Región de Murcia 2014-2020: ayudas para las medidas de agroambiente y clima y agricultura ecológica

A finales del año 2013 se inició en la Unión Europea el nuevo período de programación de desarrollo rural 2014-2020, cuyo marco normativo está constituido por un conjunto de disposiciones comunitarias a cuyo frente se sitúa el Reglamento 1305/2013 del Parlamento Europeo y del Consejo, de 17 de diciembre de 2013, relativo a la ayuda al desarrollo rural a través del Fondo Europeo Agrícola de Desarrollo Rural (FEADER). El Reglamento estructura las distintas medidas de desarrollo rural que pueden cofinanciarse a través del mencionado Fondo, entre las que destacan las destinadas al agroambiente y clima y a la agricultura ecológica ${ }^{12}$. De acuerdo con esto, la Consejería de Agricultura y Agua ha propuesto a la Comisión Europea el Programa de Desarrollo Rural de la Región de Murcia, donde se incluyen medidas relativas al agroambiente y clima y a la agricultura ecológica.

La regulación de tales ayudas en el territorio de la Región de Murcia corresponde en exclusiva a la Comunidad Autónoma — ante la ausencia de normativa estatal—, que dicta la Orden de 25 de mayo de 2015, de la Consejería de Agricultura y Agua, por la que se establecen, en la Región de Murcia, las bases reguladoras de las líneas de ayuda correspondientes a las medidas "Agroambiente y clima” y "Agricultura ecológica” del

\footnotetext{
${ }^{11}$ Resolución de 5 de mayo, de la Dirección General de Medio Ambiente, por la que se amplía para el año 2015 el periodo de peligro y se suspende la vigencia y efectos de las autorizaciones para quemas emitidas de conformidad con la Orden de 24 de mayo de 2010, de la Consejería de Agricultura y Agua, sobre medidas de prevención de incendios forestales en la Región de Murcia para el año 2010 (BORM, núm. 111 , de 16 de mayo de 2015).

12 Artículo 28 ("Agroambiente y clima") y artículo 29 (“Agricultura ecológica") del Reglamento 1305/2013 del Parlamento Europeo y del Consejo, de 17 de diciembre de 2013, relativo a la ayuda al desarrollo rural a través del Fondo Europeo Agrícola de Desarrollo Rural (FEADER).
} 
Programa de Desarrollo Rural de la Región de Murcia 2014-2020, y se aprueba, además, la convocatoria correspondiente al año 2015 de las citadas líneas de ayuda ${ }^{13}$. Entre las medidas en materia de agroambiente y clima destacan las dirigidas a la protección de las aves esteparias; la lucha biotecnológica; la protección del paisaje agrícola de la red Natura 2000; la trituración de la biomasa residual procedente de podas agrícolas; o la conservación de suelos y aguas. Por su parte, en materia de agricultura ecológica, las medidas se centran en la conversión y el mantenimiento de la agricultura ecológica.

B. Ayudas para la reconstitución del potencial de producción agrario

La acusada falta de precipitaciones vivida en la Región de Murcia el pasado año 2014 produjo una sequía extrema que ocasionó daños irreversibles en las estructuras de producción de las explotaciones agrícolas. Esta adversidad climática hizo peligrar la continuidad y supervivencia de muchas explotaciones cuyo suministro de agua son las precipitaciones, en especial, en el cultivo del almendro, donde se sufrieron daños superiores al 30\% de la producción regional. Ante la situación descrita, la Consejería de Agricultura y Agua arbitró medidas excepcionales, por lo que, mediante Orden de 30 de diciembre de 2014, se convocaron ayudas para la reconstitución del potencial de producción agrario como consecuencia de adversidades climáticas producidas en el año hidrológico 2014 en el marco de los programas de desarrollo rural 2007-2013 y 20142020 FEADER de la Región de Murcia ${ }^{14}$. Ahora bien, con el fin de simplificar la carga administrativa y la documentación justificativa aportada por los beneficiarios de tales ayudas, se hace necesario modificar las bases reguladoras y evitar la presentación de documentación innecesaria para la correcta tramitación del expediente. Por ello, la Consejería de Agricultura y Agua ha dictado la Orden de 28 de julio de 2015, por la que

\footnotetext{
${ }^{13}$ Orden de 25 de mayo de 2015, de la Consejería de Agricultura y Agua, por la que se establecen, en la Región de Murcia, las bases reguladoras de determinadas líneas de ayuda correspondientes a la medida 10 "Agroambiente y clima" y medida 11 "Agricultura ecológica" del Programa de Desarrollo Rural de la Región de Murcia 2014-2020, y se aprueba la convocatoria correspondiente al año 2015 de las citadas líneas de ayuda (BORM, núm. 120, de 27 de mayo de 2015).

${ }^{14}$ Orden de 30 de diciembre de 2014, de la Consejería de Agricultura y Agua, por la que se establecen las bases reguladoras y se convocan ayudas para la reconstitución del potencial de producción agrario como consecuencia de adversidades climáticas producidas en el año hidrológico 2014 en el marco de los Programas de Desarrollo Rural 2007-2013 y 2014-2020 FEADER de la Región de Murcia (BORM, núm. 6, de 9 de enero de 2015).
} 
se modifica la precitada Orden de 30 de diciembre de 2014, simplificando la ejecución, justificación y solicitud de pago de las ayudas ${ }^{15}$.

\subsection{Pesca}

A. Obtención de datos e información sobre el estado de la flota pesquera artesanal

Las cofradías de pescadores de la Región de Murcia, conscientes de la importancia que la conservación de los recursos pesqueros tiene como soporte básico de su economía, han considerado necesario disponer de información detallada, real y de calidad, acerca de las distintas pesquerías que constituyen su flota pesquera artesanal. Este tipo de información, recabada directamente del propio sector pesquero, es fundamental para la Administración, ya que en la actualidad no se dispone de datos de esta naturaleza relativos a la flota pesquera artesanal, siendo estos fundamentales para la elaboración de planes de gestión de las pesquerías y la adopción de medidas encaminadas a la determinación del rendimiento máximo sostenible de estas.

Por lo tanto, visto el interés público de esta información, el Consejo de Gobierno aprobó mediante Decreto 32/2015, de 23 de marzo, las normas reguladoras de una subvención a otorgar a las cofradías de pescadores de la Región de Murcia para la realización de trabajos de recopilación de datos e información de la flota pesquera artesanal de la Región de Murcia $^{16}$. Asimismo, mediante Resolución de 13 de abril de 2015, la Consejería de Agricultura y Agua publicó el convenio de colaboración entre la Comunidad Autónoma de la Región de Murcia, a través de la Consejería de Agricultura y Agua, y las cofradías de pescadores de la Región de Murcia para la obtención de datos e información sobre el estado de la flota pesquera artesanal ${ }^{17}$.

\footnotetext{
${ }^{15}$ Orden de 28 de julio de 2015, por la que se modifica la Orden de 30 de diciembre de 2014, de la Consejería de Agricultura y Agua, por la que se establecen las bases reguladoras y se convocan ayudas para la reconstitución del potencial de producción agrario como consecuencia de adversidades climáticas producidas en el año hidrológico 2014 en el marco de los Programas de Desarrollo Rural 2007-2013 y 2014-2020 FEADER de la Región de Murcia (BORM, núm. 179, de 5 de agosto de 2015).

${ }^{16}$ Decreto 32/2015, de 13 de marzo, por el que se aprueban las normas especiales reguladoras de una subvención a otorgar por concesión directa a las cofradías de pescadores de la Región de Murcia para la recogida de datos e información de la flota pesquera artesanal (BORM, núm. 63, de 17 de marzo de 2015).

17 Resolución de 13 de abril de 2015, por la que se publica el convenio de colaboración entre la Comunidad Autónoma de la Región de Murcia, a través de la Consejería de Agricultura y Agua, y las cofradías de pescadores de la Región de Murcia para la obtención de datos e información sobre el estado de la flota pesquera artesanal (BORM, núm. 93, de 24 de abril de 2015).
} 
B. Horario especial de calamento de los artes de pesca en las zonas delimitadas para el baño en el Mar Menor

Desde mediados de la década de 1990 es costumbre acotar franjas de litoral en el Mar Menor mediante corrales de red, delimitando zonas de baño, al objeto de proteger a los bañistas de la proliferación masiva de medusas que invaden estas aguas en la época estival. Sin embargo, esta delimitación de las zonas de baño coincide con la ubicación de determinados artes de pesca. Por ello, a fin de armonizar los usos de las zonas de baño y proteger la actividad pesquera en esta época, evitando la presencia de artes de pesca en las horas habituales de baño pero sin dejar de recoger las capturas de especies objetivo - que en esta época contribuyen en gran medida a la economía pesquera anual—, se hace necesario establecer, para el período estival 2015, una regulación horaria de calamento de artes en el interior de las zonas que, como consecuencia de la colocación de las redes antimedusas, quedan delimitadas para el baño.

Para ello, la Consejería de Agricultura y Agua dicta la Orden de 24 de junio de 2015, por la que se establece un horario especial de calamento de los artes de pesca en las zonas delimitadas para el baño en el Mar Menor ${ }^{18}$. Su objetivo principal es la regulación de los horarios, durante el período comprendido entre el 30 de junio y el 30 de septiembre de 2015, en el interior de las zonas de baño que hayan sido delimitadas por redes de protección frente a las medusas. La Orden de 24 de junio de 2015 fija un horario de calamento (art. 2) y un descanso semanal (art. 3), e identifica los artes (art. $4)$.

\subsection{Caza}

La Ley 7/2003, de 12 de noviembre, de Caza y Pesca Fluvial de la Región de Murcia, prevé de forma expresa la posible concesión de autorizaciones excepcionales y medidas extraordinarias por las que se puedan dejar sin efecto las prohibiciones relativas a la protección y conservación de los recursos y hábitats cinegéticos y piscícolas. En concreto, estas se concederán cuando se trate de prevenir perjuicios importantes a los cultivos, el ganado, los bosques, la pesca o la calidad de las aguas, o para proteger la

\footnotetext{
${ }^{18}$ Orden de 24 de junio de 2015, de la Consejería de Agricultura y Agua, por la que se establece un horario especial de calamento de los artes de pesca en las zonas delimitadas para el baño en el Mar Menor (BORM, núm. 148, de 30 de junio de 2015).
} 
flora o la fauna ${ }^{19}$. De acuerdo con esto, el pasado 28 de septiembre de 2015 la Dirección General de Desarrollo Rural y Forestal dictó una resolución por la que se establecen las prescripciones técnicas para las autorizaciones de control y captura de determinadas especies por daños a la agricultura y/o ganadería en terrenos cinegéticos y no cinegéticos $^{20}$. La Resolución establece un total de 23 objetivos y condiciones generales; regula el sistema de solicitud del control o captura, y la información suministrada por los agentes medioambientales para permitir la autorización; prevé una autorización directa de caza o captura con sacrificio por daños en terrenos cinegéticos y no cinegéticos, así como una autorización directa para la captura en vivo y transporte por daños en terrenos cinegéticos y no cinegéticos; identifica las especies autorizadas, los métodos de caza y captura en vivo y el período autorizable; establece una ficha de control numérico de caza o captura en vivo de especies cinegéticas por daños en terrenos cinegéticos y no cinegéticos; y prevé la suspensión de la autorización en caso de incumplimiento de alguna de las condiciones especificadas, quedando sin efecto y conllevando la incoación del pertinente expediente sancionador por infracción, en su caso, prevista en la Ley 7/2003, de 12 de noviembre, de Caza y Pesca Fluvial de la Región de Murcia.

\subsection{Sanidad animal}

Según las estadísticas de la Organización Mundial de la Salud, el norte de África es una de las zonas del mundo donde la enfermedad de la rabia se encuentra de forma endémica, no siendo infrecuente, en Europa, la aparición de casos en cánidos procedentes de Marruecos. Por otro lado, la globalización a la que el mundo está sometido y los informes epidemiológicos emitidos por diversos organismos internacionales (OIE/OMS) muestran un continuo avance de la rabia en la UE, tal y como evidencia la reiterada aparición de casos de rabia salvaje en países como Italia, Rumanía, Polonia y Eslovaquia. Finalmente, la aparición de un foco de rabia en Toledo en 2013 y la muerte en junio del 2014 de una mujer residente en Madrid que se contagió

\footnotetext{
${ }^{19}$ Artículos 52 c) y h), 53 y 55.2 de la Ley 7/2003, de 12 de noviembre, de Caza y Pesca Fluvial de la Región de Murcia.

${ }^{20}$ Resolución de la Dirección General de Desarrollo Rural y Forestal, de 28 de septiembre de 2015, por la que se establecen las prescripciones técnicas para las autorizaciones de control y captura de determinadas especies por daños a la agricultura y/o ganadería en terrenos cinegéticos y no cinegéticos. Expte. 155/2015/Caza (BORM, núm. 234, de 9 de octubre de 2015).
} 
de la enfermedad tras la mordedura de un perro en Marruecos, han puesto de manifiesto la importancia de la vacunación anual de los animales de compañía como una pieza fundamental en el control de esta enfermedad.

Por ello, con el fin de prevenir la aparición de casos de rabia humana, es necesaria la aplicación de medidas de profilaxis vacunal contra esta enfermedad en la población animal que pueda actuar como transmisora (cánidos, felinos y mustélidos). Se trata de una medida prioritaria en la lucha contra esta enfermedad para mantener un nivel adecuado de inmunización en los animales que conviven en el entorno humano. De acuerdo con esto, las consejerías de Agricultura y Agua y de Sanidad y Política Social han dictado, de forma conjunta, la Orden de 24 de junio de 2015, para el control y erradicación de la rabia en los animales y la prevención de riesgos para la salud pública $^{21}$. Esta tiene por objeto establecer, con carácter obligatorio, la vacunación anual de rabia en los animales domésticos de hábitat humano, en concreto las especies caninas, felinas y mustélidas (hurones), ubicados en la Región de Murcia.

\section{Ordenanzas locales}

\subsection{Cieza}

A. Aprobación definitiva del Reglamento regulador del servicio público de abastecimiento de agua potable al municipio de Cieza (BORM, núm. 238, de 15 de octubre de 2015)

El Pleno del Ayuntamiento de Cieza, en sesión extraordinaria urgente celebrada el día 21 de mayo de 2015, acordó la aprobación inicial del Reglamento regulador del servicio público de abastecimiento de agua potable al municipio de Cieza, aprobado en octubre de 2015 de forma definitiva. Se trata de un reglamento compuesto por 74 artículos en los que, junto con las disposiciones generales relativas al objeto y ámbito de aplicación, la titularidad del servicio y las facultades de gestión y las definiciones generales (título I), se regulan cuestiones concernientes a los derechos y las obligaciones (título II); los elementos materiales del servicio: instalaciones generales, acometidas, ampliaciones de red o suministro en nuevas actuaciones urbanísticas (título III); aspectos del suministro

\footnotetext{
${ }^{21}$ Orden de 24 de junio de 2015, de las Consejerías de Agricultura y Agua y de Sanidad y Política Social, para el control y erradicación de la rabia en los animales y la prevención de riesgos para la salud pública (BORM, núm. 148, de 30 de junio de 2015).
} 
como su contratación, suspensión y regularidad, o la medición de los consumos (título IV); la lectura, la facturación y el cobro (título V); comprobaciones y defraudaciones (título VI); y, en último lugar, el régimen sancionador y las reclamaciones (títulos VII y VIII, respectivamente). Finalmente, el Reglamento se cierra con un título IX relativo a las medidas de ahorro de agua.

B. Aprobación definitiva de la Ordenanza reguladora del uso y vertido de aguas residuales a la red de alcantarillado (BORM, núm. 238, de 15 de octubre de 2015)

El Pleno del Ayuntamiento de Cieza, en sesión extraordinaria urgente celebrada el día 21 de mayo de 2015, acordó la aprobación inicial de la Ordenanza municipal reguladora del uso y vertido de aguas residuales a la red de alcantarillado del Ayuntamiento de Cieza. Esta ordenanza, aprobada ahora de forma definitiva, regula, junto con las disposiciones generales sobre el objeto, el ámbito de aplicación y la titularidad del vertido (título I), cuestiones relativas a las normas para el uso y la construcción de la red de alcantarillado (título II), los vertidos a la red de alcantarillado (título III) y el régimen disciplinario (título IV).

3.2. Blanca: aprobación inicial de la modificación de la Ordenanza reguladora de la intervención administrativa en la edificación y uso del suelo (BORM, núm. 217, de 19 de septiembre de 2015)

El Pleno del Ayuntamiento, en sesión celebrada el día 10 de septiembre de 2015, acordó la aprobación inicial de la modificación de la Ordenanza reguladora de la intervención administrativa en la edificación y uso del suelo. De este modo, en cumplimiento de lo dispuesto en los artículos 49 y 70.2 de la Ley 7/1985, de 2 de abril, Reguladora de las Bases del Régimen Local, se sometió el expediente a información pública por el plazo de treinta días, a contar desde el día siguiente a la inserción del anuncio en el Boletín Oficial de la Región de Murcia, tras lo cual, de no existir reclamaciones, se considera aprobado definitivamente dicho Acuerdo. 
3.3. Yecla: convocatoria de subvenciones en el ámbito de los programas de medio ambiente, ejercicio 2015 (BORM, núm. 174, de 30 de julio de 2015)

La Junta de Gobierno Local, en sesión celebrada el día 14 de julio de 2015, aprobó la convocatoria para el otorgamiento de subvenciones en el ámbito de los programas de medio ambiente, correspondientes al ejercicio 2015. Dicha convocatoria se rige por las bases publicadas en el Boletín Oficial de la Región de Murcia núm. 245, de 22 de octubre de 2013. Las bases pueden obtenerse en la Secretaría General y en la página web del Ayuntamiento de Yecla (www.yecla.es).

3.4. Lorquí: aprobación definitiva de la Ordenanza reguladora del uso y vertidos a la red de alcantarillado del municipio de Lorquí (BORM, núm. 170, de 25 de julio de 2015)

Se ha elevado a definitivo, por ausencia de reclamaciones, el acuerdo del Pleno del Ayuntamiento adoptado en sesión celebrada el día 12 de marzo de 2015, por el que se aprueba inicialmente la Ordenanza reguladora del uso y vertidos a la red de alcantarillado del municipio de Lorquí. En esta se regulan, junto con las disposiciones generales relativas al objeto, el marco normativo y el ámbito de aplicación (título I), las normas para el uso y la construcción de la red de alcantarillado (título II), los vertidos a la red de alcantarillado (título III) y, en último lugar, el régimen disciplinario (título IV).

3.5. Mazarrón: modificación de la Ordenanza municipal de uso y aprovechamiento de playas (BORM, núm. 137, de 17 de junio de 2015)

Las características del municipio de Mazarrón, situado a orillas del Mediterráneo, y su litoral, con 35 kilómetros de costa y 33 playas y calas naturales, convierten este municipio en un lugar privilegiado para el disfrute del baño en el mar, así como para la práctica de todo tipo de deportes náuticos. Por ello, se considera imprescindible la Ordenanza municipal de uso y aprovechamiento de playas, teniendo en cuenta asimismo las ordenanzas de limpieza y medio ambiente del municipio, a fin de controlar el uso, disfrute y aprovechamiento de las playas, observando todas las normas de seguridad e higiene necesarias para facilitar la convivencia de los usuarios de las zonas de baño y evitar cualquier tipo de incidentes en ellas. 
Vista su importancia, mediante Resolución de 26 de mayo de 2015 se eleva a definitivo el acuerdo del Pleno del Ayuntamiento, adoptado en sesión ordinaria del día 24 de febrero de 2015, por el que se aprueba la modificación de la Ordenanza municipal de uso y aprovechamiento de las playas. Esta ordenanza regula, junto con las disposiciones generales, la limpieza e higiene de las playas (título II); la presencia de animales en las playas (título III); la práctica de la pesca (título IV); las acampadas y la circulación de vehículos en las playas (título V); las embarcaciones y otros objetos, artefactos flotantes (título VI); la práctica de juegos (título VII); la venta ambulante (título VIII); la vigilancia de playas (título IX); la fauna y la flora (título X); las instalaciones y los elementos de temporada (título XI); y el régimen sancionador (título XII). 